\title{
Carphology by A fo Ben
}

\section{NEURO-19}

A Fo Ben appreciates that the world will have significantly moved on by the time this edition of PN hits the printers, but in an attempt to move with the times, this is a report from medRxiv (and comes with all the caveats for pre-peer-reviewed papers) - but it is highly pertinent. A study of 214 hospitlaised patients with COVID-19 from Wuhan, China, looked at the neurological features of severe and milder cases. Seventy-eight (36\%) had neurological symptoms-primarily dizziness (17\%), headache (13\% total, $17 \%$ of severe cases) and impaired consciousness levels $(7.5 \%$ total, $15 \%$ of severe cases). Fortunately, seizures were only seen in $0.5 \%$. Fortunately for me, I've been socially distancing myself long before it was cool to do so.

medRxiv 2020;02.22.20026500. JAMA Neurol 2020 doi:10.1001/jamaneurol.2020. 1127

\section{UPLIFTING STORY}

Continuing on a theme, a current concern is the lack of personal protective equipment available to frontline clinical staff. If your priority is to 'Be safe, be Sexy' (or survive the pandemic, lift and separate), look no further than the 2009 IgNobel prize winner Elena Bodnar's patent for 'inventing a brassiere that, in an emergency, can be quickly converted into a pair of protective face masks'. Or if you are looking for a heartwarming angle-one for you and one for a bystander (figure 1).

\section{READY, ESETT, GO!}

How do we best manage neurological emergencies (particularly when ITU is full)? To investigate the second stage of status epilepticus (following benzos), the ESETT team undertook a multicentre, doubleblind, response-adaptive, randomised controlled trial, and recruited patients from 58 hospital emergency departments across the USA. Adults and children (2 years and up) were recruited. Patients were randomised to receive one of intravenous levetiracetam, fosphenytoin and valproate. Children, adults and older adults with established status epilepticus responded similarly to the three drugs, with treatment success in approximately half of the patients. Intubation was more common for children randomised to fosphenytoin. It seems like it matters less what you give, provided you give enough of it and promptly-levetiracetam $60 \mathrm{mg} / \mathrm{kg}$ (maximum $4500 \mathrm{mg}$ ), fosphenytoin $20 \mathrm{mg}$ per $\mathrm{kg}$ (maximum $1500 \mathrm{mg}$ ) or valproate $40 \mathrm{mg} / \mathrm{kg}$ (maximum $3000 \mathrm{mg}$ ).

Lancet 2020;395(10231):1217-1224.

\section{MUCH MORE SERIOUS THAN \\ THAT}

Sport is thought to be a 'good thing'psychologically and physically-but could there be benefits to soccer players because of this pandemicenforced break in play? A retrospective cohort study compared mortality from neurodegenerative disease among 7676 former professional Scottish soccer players and 23028 matched controls. Mortality in players was lower for ischaemic heart disease and lung cancer-but mortality from neurodegenerative diseases was more common $(1.7 \%$ vs $0.5 \%)$. Former players were more likely to be prescribed dementia-related medications (and these were less likely to be prescribed to goalkeepers vs outfield players). Whether this hints at an increased rate of chronic traumatic encephalopathy from low-level concussion and heading heavy wet leather balls is unproven. All of this puts Bill Shankly's 'Some people think football is a matter of life and death. I assure you, it's much more serious than that' into sharp relief.

N Engl J Med 2019;381(19):1801-8.

Competing interests None declared.

Provenance and peer

review Commissioned; internally peer reviewed.

Patient consent for publication Not required.
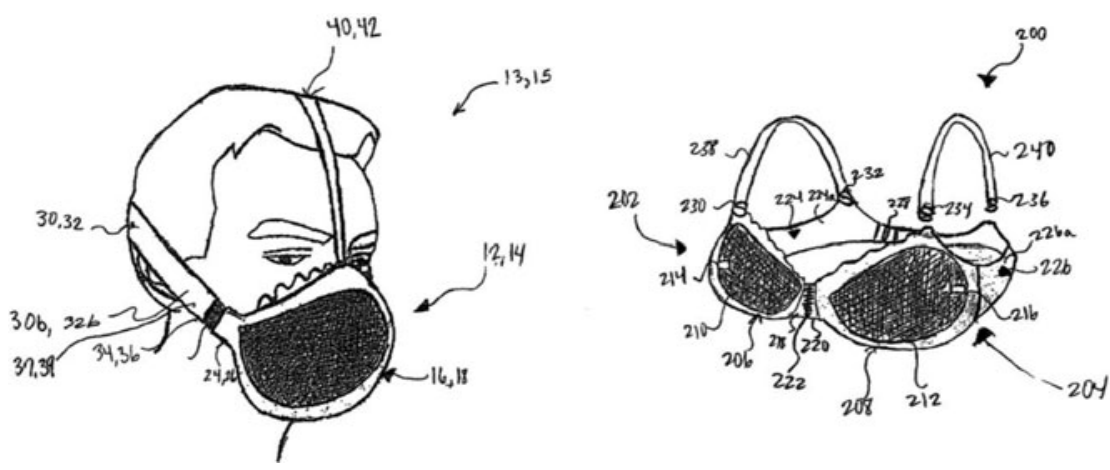

Figure 1 Taken from US patent US7255627B2. 